\title{
The Modified Simple Equation Method for Exact and Solitary Wave Solutions of Nonlinear Evolution Equation: The GZK-BBM Equation and Right-Handed Noncommutative Burgers Equations
}

\author{
Kamruzzaman Khan, ${ }^{1}$ M. Ali Akbar, ${ }^{2,3}$ and Norhashidah Hj. Mohd. Ali ${ }^{3}$ \\ ${ }^{1}$ Department of Mathematics, Pabna Science and Technology University, Pabna 6600, Bangladesh \\ ${ }^{2}$ Department of Applied Mathematics, University of Rajshahi, Rajshahi 6205, Bangladesh \\ ${ }^{3}$ School of Mathematical Sciences, Universiti Sains Malaysia, 11800 USM Pulau Pinang, Malaysia \\ Correspondence should be addressed to M. Ali Akbar; ali_math74@yahoo.com
}

Received 25 November 2012; Accepted 10 January 2013

Academic Editors: A. Herrera-Aguilar, W.-H. Steeb, and H. Zhou

Copyright (C) 2013 Kamruzzaman Khan et al. This is an open access article distributed under the Creative Commons Attribution License, which permits unrestricted use, distribution, and reproduction in any medium, provided the original work is properly cited.

The modified simple equation method is significant for finding the exact traveling wave solutions of nonlinear evolution equations (NLEEs) in mathematical physics. In this paper, we bring in the modified simple equation (MSE) method for solving NLEEs via the Generalized Zakharov-Kuznetsov-Benjamin-Bona-Mahony (GZK-BBM) equation and the right-handed noncommutative Burgers' (nc-Burgers) equations and achieve the exact solutions involving parameters. When the parameters are taken as special values, the solitary wave solutions are originated from the traveling wave solutions. It is established that the MSE method offers a further influential mathematical tool for constructing the exact solutions of NLEEs in mathematical physics.

\section{Introduction}

The importance of nonlinear evolution equations (NLEEs) is now well established, since these equations arise in various areas of science and engineering, especially in fluid mechanics, biology, plasma physics, solid-state physics, optical fibers, biophysics and so on. As a key problem, finding their analytical solutions is of great importance and is actually executed through various efficient and powerful methods such as the Exp-function method [1-4], the tanh-function method $[5,6]$, the homogeneous balance method $[7,8]$, the $\left(G^{\prime} / G\right)$-expansion method [9-16], the Hirota's bilinear transformation method [17, 18], the Backlund transformation method [19], the inverse scattering transformation [20], the Jacobi elliptic function method [21], the modified simple equation method [22-24] and so on.

The objective of this paper is to look for new study relating to the MSE method via the well-recognized GZK-BBM equation and right-handed nc-Burgers' equation and establish the originality and effectiveness of the method.

The paper is organized as follows: in Section 2, we give the description of the MSE method. In Section 3, we use this method to the nonlinear evolution equations pointed out above, and in Section 4 conclusions are given.

\section{Description of the MSE Method}

Suppose the nonlinear evolution equation is in the following form:

$$
F\left(u, u_{t}, u_{x}, u_{x x}, u_{t t}, \ldots\right)=0,
$$

where $F$ is a polynomial of $u(x, t)$ and its partial derivatives wherein the highest order derivatives and nonlinear terms are concerned. The main steps of the MSE method [22-24] are as follows. 
Step 1. The traveling wave transformation

$$
u(x, t)=u(\xi), \quad \xi=x \pm v t
$$

permits us to reduce (1) into the following ordinary differential equation $(\mathrm{ODE})$ :

$$
P\left(u, u^{\prime}, u^{\prime \prime}, \ldots\right)=0
$$

where $P$ is a polynomial in $u(\xi)$ and its total derivatives, wherein $u^{\prime}(\xi)=d u / d \xi$.

Step 2. We suppose the solution of (3) is of the form

$$
u(\xi)=\sum_{k=0}^{N} C_{k}\left[\frac{\Phi^{\prime}(\xi)}{\Phi(\xi)}\right]^{k}
$$

where $C_{k}(k=0,1,2,3, \ldots)$ are arbitrary constants to be determined, such that $C_{N} \neq 0$, and $\Phi(\xi)$ is an unidentified function to be determined afterwards. In Exp-function method, $\left(G^{\prime} / G\right)$-expansion method, tanh-function method, Jacobi elliptic function method, and so forth, the solution is offered in terms of some predefined functions, but in the MSE method, $\Phi$ is not predefined or not a solution of any predefined differential equation. Therefore, some fresh solutions might be found by this method. This is the merit of the MSE method.

Step 3. We determine the positive integer $N$ come out in (4) by considering the homogeneous balance between the highest order derivatives and the highest order nonlinear terms occurring in (3).

Step 4. We compute all the required derivatives $u^{\prime}, u^{\prime \prime}, \ldots$, and substitute (4) and the derivatives into (3) and then we account for the function $\Phi(\xi)$. As a result of this substitution, we get a polynomial of $\left(\Phi^{\prime}(\xi) / \Phi(\xi)\right)$ and its derivatives. In this polynomial, we equate all the coefficients to zero. This procedure yields a system of equations whichever can be solved to find $C_{k}$ and $\Phi(\xi)$.

\section{Applications}

3.1. The GZK-BBM Equation. In this subsection, we will use the MSE method to look for the exact solutions and then the solitary wave solutions to the GZK-BBM equation:

$$
u_{t}+u_{x}+\alpha\left(u^{3}\right)_{x}+\beta\left(u_{x t}+u_{y y}\right)_{x}=0
$$

where $\alpha$ and $\beta$ are nonzero constants.

Using traveling wave transformation,

$$
u=u(x, y, t), \quad \xi=x+y-v t, \quad u(x, y, t)=u(\xi),
$$

(5) reduces to the following ODE:

$$
-v u^{\prime}+u^{\prime}+\alpha\left(u^{3}\right)^{\prime}+\beta\left(-v u^{\prime \prime}+u^{\prime \prime}\right)^{\prime}=0 .
$$

Integrating (7) with respect to $\xi$, we obtain

$$
(1-v) u+\alpha u^{3}+\beta(1-v) u^{\prime \prime}=0
$$

Balancing the highest order derivative $u^{\prime \prime}$ and nonlinear term $u^{3}$, we obtain $N=1$.

Therefore, the solution (4) turns into the following form:

$$
u(\xi)=C_{0}+C_{1}\left(\frac{\Phi^{\prime}}{\Phi}\right)
$$

where $C_{0}$ and $C_{1}$ are constants such that $C_{1} \neq 0$, and $\Phi(\xi)$ is an unidentified function to be determined. It is easy to find that

$$
\begin{gathered}
u^{\prime}=C_{1}\left[\frac{\Phi^{\prime \prime}}{\Phi}-\left(\frac{\Phi^{\prime}}{\Phi}\right)^{2}\right] \\
u^{\prime \prime}=C_{1} \frac{\Phi^{\prime \prime \prime}}{\Phi}-3 C_{1} \frac{\Phi^{\prime \prime} \Phi^{\prime}}{\Phi^{2}}+2 A_{1}\left(\frac{\Phi^{\prime}}{\Phi}\right)^{3} \\
u^{3}=C_{1}{ }^{3}\left(\frac{\Phi^{\prime}}{\Phi}\right)^{3}+3 C_{1}{ }^{2} C_{0}\left(\frac{\Phi^{\prime}}{\Phi}\right)^{2}+3 C_{1} C_{0}{ }^{2}\left(\frac{\Phi^{\prime}}{\Phi}\right)+C_{0}{ }^{3} .
\end{gathered}
$$

Substituting the values of $u, u^{\prime \prime}$, and $u^{3}$ from (9)-(12) into (8) and then equating the coefficients of $\Phi^{0}, \Phi^{-1}, \Phi^{-2}$, and $\Phi^{-3}$ to zero, we obtain

$$
\begin{gathered}
\alpha C_{0}{ }^{3}+C_{0}-v C_{0}=0, \\
\beta(v-1) \Phi^{\prime \prime \prime}-\left(3 \alpha C_{0}{ }^{2}-v+1\right) \Phi^{\prime}=0, \\
\beta(v-1) \Phi^{\prime \prime}+\alpha C_{0} C_{1} \Phi^{\prime}=0, \\
\left(\alpha C_{1}{ }^{3}+2 \beta C_{1}-2 \beta v C_{1}\right)\left(\Phi^{\prime}\right)^{3}=0 .
\end{gathered}
$$

Equations (13) and (16), respectively, yield

$$
C_{0}=0, \pm \sqrt{\frac{\nu-1}{\alpha}}, \quad C_{1}= \pm \sqrt{\frac{2 \beta(\nu-1)}{\alpha}} \text { since } C_{1} \neq 0 \text {, }
$$

where $v \neq 1$.

From (14) and (15), we obtain

$$
\frac{\Phi^{\prime \prime \prime}}{\Phi^{\prime \prime}}=-l
$$

where $l=\left(3 \alpha C_{0}^{2}-v+1\right) / \alpha C_{0} C_{1}$.

Integrating (18), we obtain

$$
\Phi^{\prime \prime}(\xi)=c_{1} e^{-l \xi},
$$

where $c_{1}$ is a constant of integration.

And from (15) and (19), we obtain

$$
\Phi^{\prime}=-m e^{-l \xi},
$$

where $m=\beta(v-1) c_{1} / \alpha C_{0} C_{1}$ 
Integrating (20) with respect to $\xi$, we obtain

$$
\Phi(\xi)=c_{2}+\frac{m}{l} e^{-l \xi}
$$

where $c_{2}$ is a constant of integration.

Substituting the value of $\Phi$ and $\Phi^{\prime}$ into solution (9) yields

$$
u(\xi)=C_{0}+C_{1}\left(\frac{-l m e^{-l \xi}}{c_{2} l+m e^{-l \xi}}\right) .
$$

Case 1. When $C_{0}=0$, solution (22) collapses, and hence this case is rejected.

Case 2. When $C_{0}= \pm \sqrt{(\nu-1) / \alpha}$ and $C_{1}= \pm \sqrt{2 \beta(v-1) / \alpha}$, substituting the values of $C_{0}$ and $C_{1}$ into (22) and simplifying, we obtain the exact solutions:

$$
\begin{aligned}
u(x, y, t)= & \pm \sqrt{\left(\frac{v-1}{\alpha}\right)} \\
& \times\left\{1-\frac{2 \beta c_{1} \exp ( \pm \sqrt{2 / \beta}(x+y-v t))}{\beta c_{1} \exp ( \pm \sqrt{2 / \beta}(x+y-v t))+2 c_{2}}\right\} .
\end{aligned}
$$

Since $c_{1}$ and $c_{2}$ are arbitrary constants, therefore, if we set $c_{2}=$ $c_{1} / 2$, the exact solution (23) turns out to the following solitary wave solutions

$$
u(x, y, t)= \pm \sqrt{\left(\frac{v-1}{\alpha}\right)} \tanh \left(\sqrt{\frac{1}{2}}(x+y-v t)\right)
$$

when $\beta=1$. On the other hand, if $\beta=-1$, solution (23) turns into

$$
u(x, y, t)= \pm \sqrt{\left(\frac{v-1}{\alpha}\right)} \operatorname{coth}\left(\sqrt{\frac{1}{2}}(x+y-v t)\right) .
$$

In particular, when $\alpha=1$ and $v=4$, solutions (24) and (25) convert to

$$
\begin{aligned}
& u(x, y, t)= \pm \sqrt{3} \tanh \left(\sqrt{\frac{1}{2}}(x+y-4 t)\right), \\
& u(x, y, t)= \pm \sqrt{3} \operatorname{coth}\left(\sqrt{\frac{1}{2}}(x+y-4 t)\right),
\end{aligned}
$$

respectively.

For $y=0$, the solution $u(x, y, t)$ presented in (26) is sketched in Figure 1.

Again for $y=0$, the solution $u(x, y, t)$ presented in (27) is sketched in Figure 2.

The MSE method is applied to investigate solitary wave solutions to the GZK-BBM equation and obtained solutions with free parameters involving the known solutions in the open literature. Obviously we might choose the values of the arbitrary constants $c_{1}$ and $c_{2}$ equal to other values, resulting in diverse solitary shapes. The free parameters imply some physical meaningful results in gravity water waves in the long-wave regime.

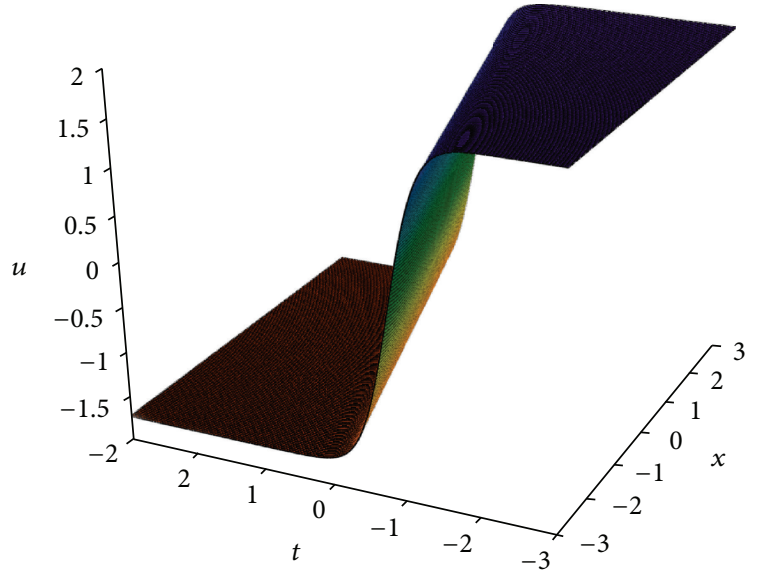

Figure 1

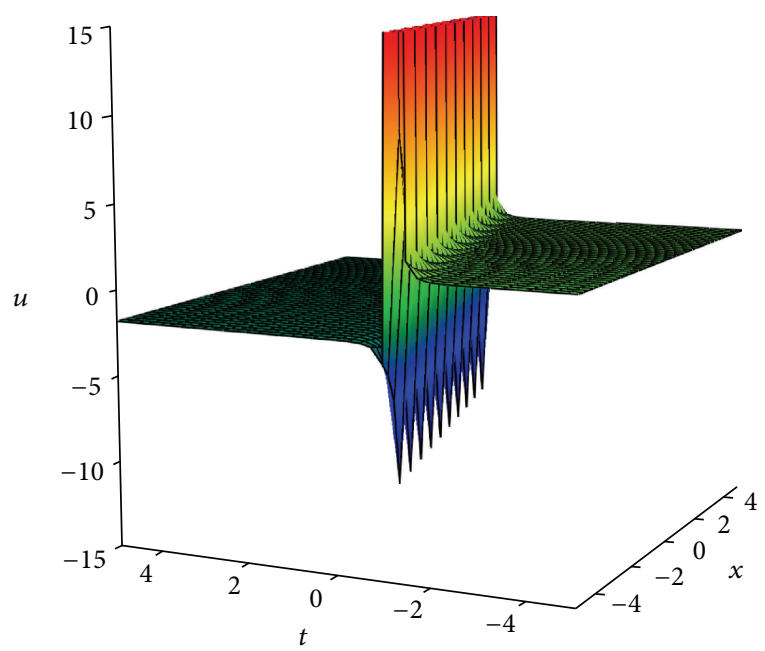

Figure 2

3.2. The Right-Handed nc-Burgers' Equation. In this subsection, we will bring to bear the MSE method to find the traveling wave solutions and then the solitary wave solutions to the right-handed nc-Burgers' equation:

$$
u_{t}=u_{x x}+2 u u_{x}
$$

Using traveling wave transformation (2), (28) is reduced to the following ODE:

$$
u^{\prime \prime}+2 u u^{\prime}+v u^{\prime}=0 .
$$

Integrating (29) with respect to $\xi$ and setting the constant of integration to zero, we obtain

$$
u^{\prime}+u^{2}+v u=0 \text {. }
$$

Balancing the highest order derivative and nonlinear term, we obtain $N=1$.

Therefore, solution (4) becomes

$$
u(\xi)=C_{0}+C_{1}\left(\frac{\Phi^{\prime}}{\Phi}\right)
$$


Executing the parallel course of action which is described in Section 3.1, we obtain

$$
\begin{gathered}
v C_{0}+C_{0}{ }^{2}=0, \\
\Phi^{\prime \prime}+\left(2 C_{0}+v\right) \Phi^{\prime}=0, \\
C_{1}^{2}-C_{1}=0 .
\end{gathered}
$$

Solving (32) and (34), we obtain $C_{0}=0,-v$ and $C_{1}=1$, since $C_{1} \neq 0$, respectively.

Case 1. When $C_{0}=0$ and $C_{1}=1$ and solving (33), we receive the value of $\Phi$, and substituting the value of $\Phi$ into (31), we obtain the following exact solution:

$$
u(x, t)=\frac{v c_{1} \exp (-v(x-v t))}{v c_{2}-c_{1} \exp (-v(x-v t))},
$$

where $c_{1}$ and $c_{2}$ are constants of integration. Therefore, we can make choices at random the parameters $c_{1}$ and $c_{2}$; if we choose $c_{1}=v$ and $c_{2}=1$, the exact solution (35) turns into the under determined solitary wave solution

$$
u(x, t)=\frac{-v}{2}\left\{1-\operatorname{coth}\left(v \frac{(x-v t)}{2}\right)\right\} .
$$

And if $c_{1}=-v$ and $c_{2}=1$, the solution (35) turn into,

$$
u(x, t)=\frac{-v}{2}\left\{1-\tanh \left(v \frac{(x-v t)}{2}\right)\right\} .
$$

Case 2. When $C_{0}=-v$ and solving (33), we get the value of $\Phi$, and substituting this value into (31), we obtain the subsequent exact solution:

$$
u(x, t)=-v+\frac{v c_{1} \exp (v(x-v t))}{c_{1} \exp (v(x-v t))+v c_{2}} .
$$

We can arbitrarily pick the parameters $c_{1}$ and $c_{2}$. Therefore, exact solution (38) turns into the following solitary wave solutions:

$$
u(x, t)=-v+\frac{v}{2}\left\{1+\tanh \left(v \frac{(x-v t)}{2}\right)\right\},
$$

when $c_{1}=v$ and $c_{2}=1$, and

$$
u(x, t)=-v+\frac{v}{2}\left\{1+\operatorname{coth}\left(\frac{v(x-v t)}{2}\right)\right\}
$$

when $c_{1}=-v$ and $c_{2}=1$.

The solution $u(x, t)$ given in (39) is presented in Figure 3. The solution $u(x, t)$ given in (40) is presented in Figure 4.

For specific values of the parameters in the generalized exact solutions (35) and (38), we obtain the solitary wave shape solutions to the right-handed nc-Burgers' equation which are shown in Figures 3 and 4. Of course we might choose other values of the arbitrary constants $c_{1}$ and $c_{2}$, resulting in diverse solitary wave shapes. The free parameters may imply some physical meaningful results in fluid mechanics, gas dynamics, and traffic flow.

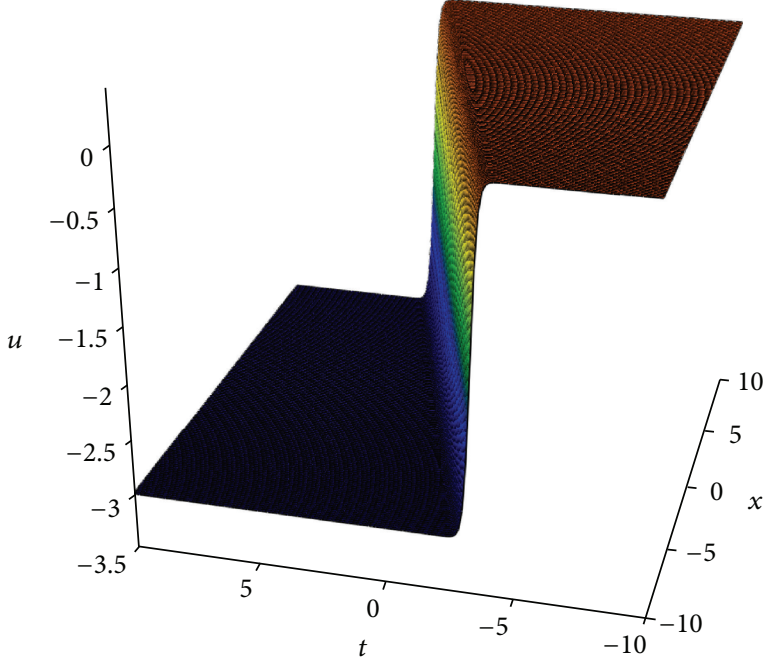

FiguRE 3

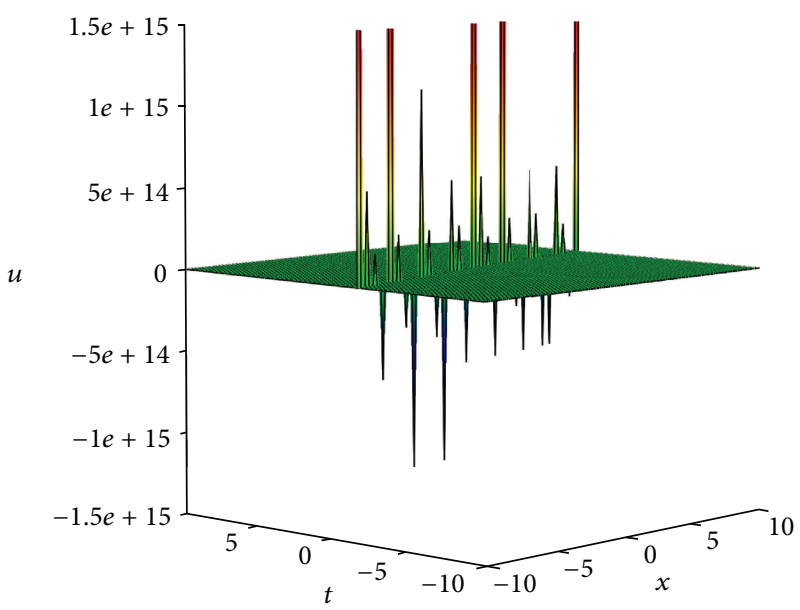

FIGURE 4

\section{Conclusions}

The modified simple equation method presented in this paper has been successfully implemented to find the exact and the solitary wave solutions for NLEEs via the GZK-BBM and right-handed nc-Burgers' equation. The method offers solutions with free parameters that might be important to explain some intricate physical phenomena. Some special solutions including the known solitary wave solution are originated by setting appropriate values for the parameters. Compared to the currently proposed method with other methods, such as the $\left(G^{\prime} / G\right)$-expansion method, the Exp-function method and the tanh-function method, we might conclude that the exact solutions to (5) and (28) can be investigated using these methods with the help of the symbolic computational software such as Mathematica and Maple to facilitate the complex algebraic computations. On the other hand, via the proposed method, the exact and solitary wave solutions to these equations have been achieved without using any 
symbolic computation software because the method is very simple and has easy computations.

\section{References}

[1] J. H. He and X. H. Wu, "Exp-function method for nonlinear wave equations," Chaos, Solitons \& Fractals, vol. 30, no. 3, pp. 700-708, 2006.

[2] M. A. Akbar and N. H. M. Ali, "Exp-function method for duffing equation and new solutions of $(2+1)$ dimensional dispersive long Wave Equations," Progress in Applied Mathematics, vol. 1, no. 2, pp. 30-42, 2011.

[3] H. Naher, F. A. Abdullah, and M. Ali Akbar, "New traveling wave solutions of the higher dimensional nonlinear partial differential equation by the exp-function method," Journal of Applied Mathematics, Article ID 575387, 14 pages, 2012.

[4] H. Naher, A. F. Abdullah, and M. A. Akbar, "The Exp-function method for new exact solutions of the nonlinear partial differential equations," International Journal of the Physical Sciences, vol. 6, no. 29, pp. 6706-6716, 2011.

[5] M. A. Abdou, "The extended tanh method and its applications for solving nonlinear physical models," Applied Mathematics and Computation, vol. 190, no. 1, pp. 988-996, 2007.

[6] E. Fan, "Extended tanh-function method and its applications to nonlinear equations," Physics Letters A, vol. 277, no. 4-5, pp. 212-218, 2000.

[7] M. L. Wang, "Solitary wave solutions for variant Boussinesq equations," Physics Letters A, vol. 199, no. 3-4, pp. 169-172, 1995.

[8] E. M. E. Zayed, H. A. Zedan, and K. A. Gepreel, "On the solitary wave solutions for nonlinear Hirota-Satsuma coupled KdV of equations," Chaos, Solitons \& Fractals, vol. 22, no. 2, pp. 285303, 2004.

[9] M. Wang, X. Li, and J. Zhang, “The $\left(G^{\prime} / G\right)$-expansion method and travelling wave solutions of nonlinear evolution equations in mathematical physics," Physics Letters A, vol. 372, no. 4, pp. 417-423, 2008.

[10] E. M. E. Zayed and K. A. Gepreel, "The $\left(G^{\prime} / G\right)$-expansion method for finding traveling wave solutions of nonlinear partial differential equations in mathematical physics," Journal of Mathematical Physics, vol. 50, no. 1, pp. 013502-013514, 2009.

[11] E. M. E. Zayed, "Traveling wave solutions for higher dimensional nonlinear evolution equations using the $\left(G^{\prime} / G\right)$ expansion method," Journal of Applied Mathematics \& Informatics, vol. 28, pp. 383-395, 2010.

[12] M. A. Akbar, N. H. M. Ali, and E. M. E. Zayed, "A generalized and improved $\left(G^{\prime} / G\right)$-expansion method for nonlinear evolution equations," Mathematical Problems in Engineering, vol. 2012, Article ID 459879, 22 pages, 2012.

[13] M. Ali Akbar, N. Hj. Mohd. Ali, and E. M. E. Zayed, "Abundant exact traveling wave solutions of generalized Bretherton equation via $\left(G^{\prime} / G\right)$-expansion method," Communications in Theoretical Physics, vol. 57, no. 2, pp. 173-178, 2012.

[14] M. A. Akbar, N. H. M. Ali, and S. T. Mohyud-Din, "The alternative $\left(G^{\prime} / G\right)$-expansion method with generalized Riccati equation: application to fifth order $(1+1)$-dimensional CaudreyDodd-Gibbon equation," International Journal of Physical Sciences, vol. 7, no. 5, pp. 743-752, 2012.

[15] M. A. Akbar and N. H. M. Ali, “The alternative $\left(G^{\prime} / G\right)$ expansion method and its applications to nonlinear partial differential equations," International Journal of Physical Sciences, vol. 6, no. 35, pp. 7910-7920, 2011.

[16] M. A. Akbar, N. H. M. Ali, and S. T. Mohyud-Din, "Some new exact traveling wave solutions to the $(3+1)$-dimensional Kadomtsev-Petviashvili equation," World Applied Sciences Journal, vol. 16, no. 11, pp. 1551-1558, 2012.

[17] R. Hirota, "Exact envelope-soliton solutions of a nonlinear wave equation," Journal of Mathematical Physics, vol. 14, pp. 805-809, 1973.

[18] R. Hirota and J. Satsuma, "Soliton solutions of a coupled Korteweg-de Vries equation," Physics Letters A, vol. 85, no. 8-9, pp. 407-408, 1981.

[19] M. R. Miura, Backlund Transformation, Springer, Berlin, Germany, 1978.

[20] M. J. Ablowitz and P. A. Clarkson, Solitons, nonlinear evolution equations and inverse scattering, vol. 149 of London Mathematical Society Lecture Note Series, Cambridge University Press, Cambridge, UK, 1991.

[21] D. Lu and Q. Shi, "New Jacobi elliptic functions solutions for the combined KdV-MKdV equation," International Journal of Nonlinear Science, vol. 10, no. 3, pp. 320-325, 2010.

[22] A. J. M. Jawad, M. D. Petković, and A. Biswas, "Modified simple equation method for nonlinear evolution equations," Applied Mathematics and Computation, vol. 217, no. 2, pp. 869-877, 2010.

[23] E. M. E. Zayed, "A note on the modified simple equation method applied to Sharma-Tasso-Olver equation," Applied Mathematics and Computation, vol. 218, no. 7, pp. 3962-3964, 2011.

[24] E. M. E. Zayed and S. A. H. Ibrahim, "Exact solutions of nonlinear evolution equations in mathematical physics using the modified simple equation method," Chinese Physics Letters, vol. 29, no. 6, Article ID 060201, 2012. 


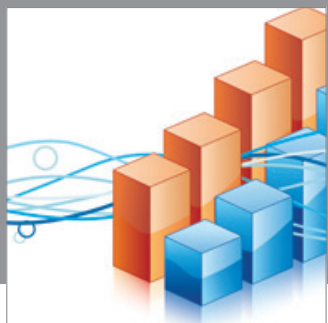

Advances in

Operations Research

mansans

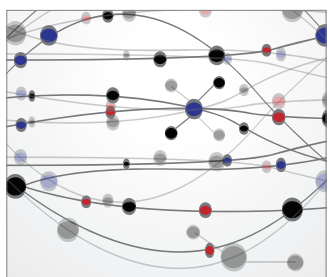

The Scientific World Journal
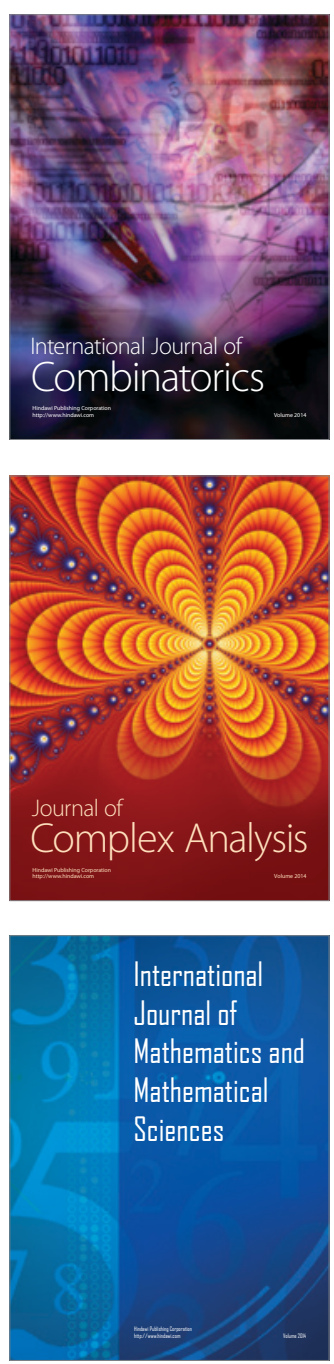
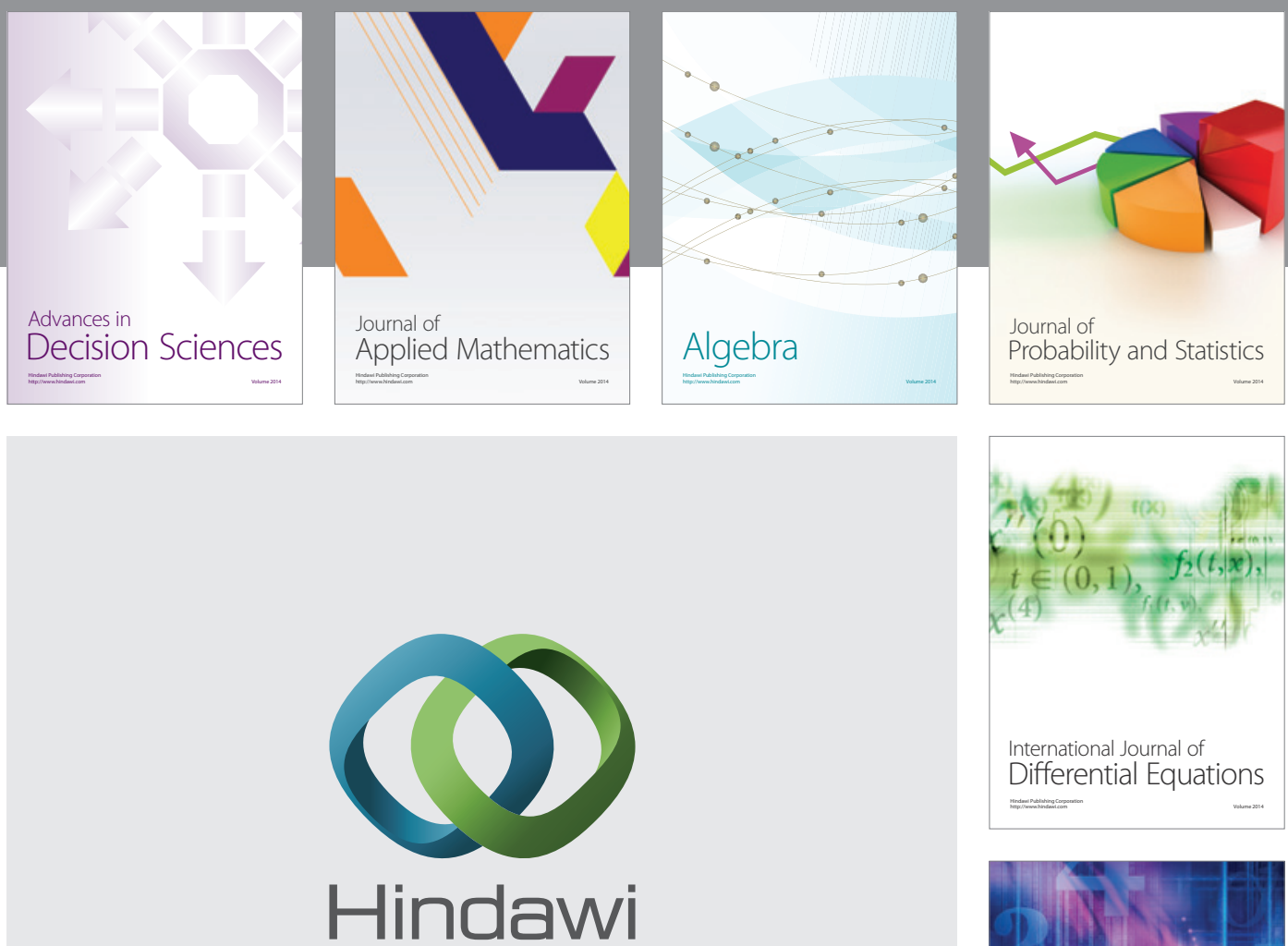

Submit your manuscripts at http://www.hindawi.com
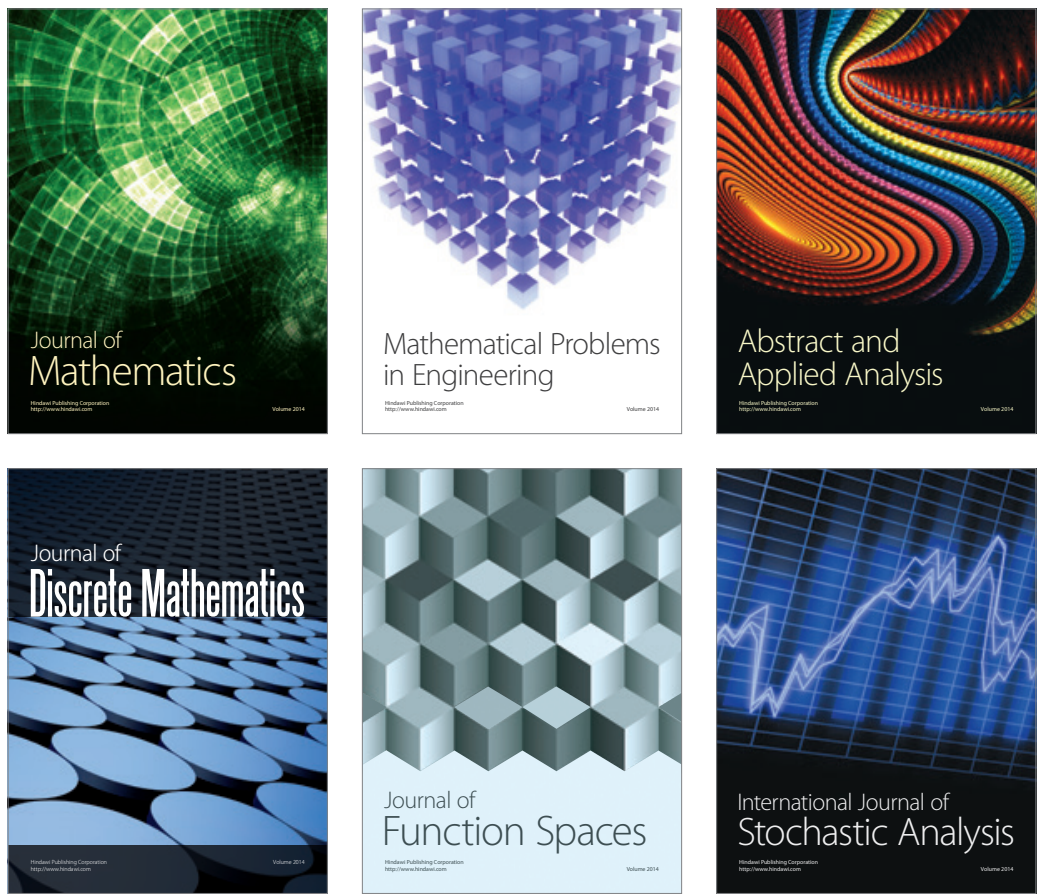

Journal of

Function Spaces

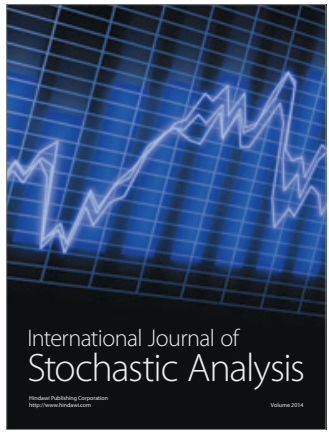

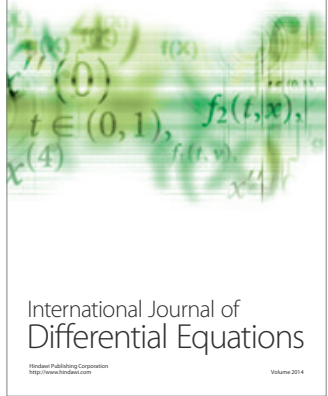
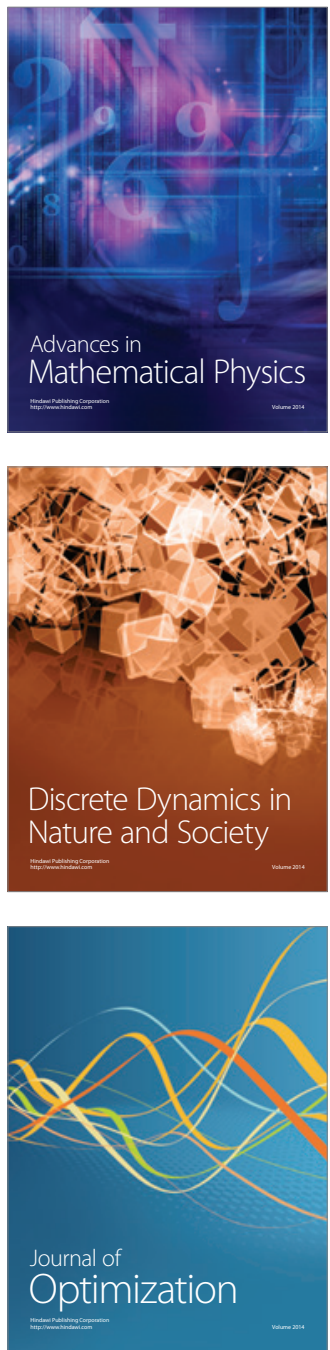